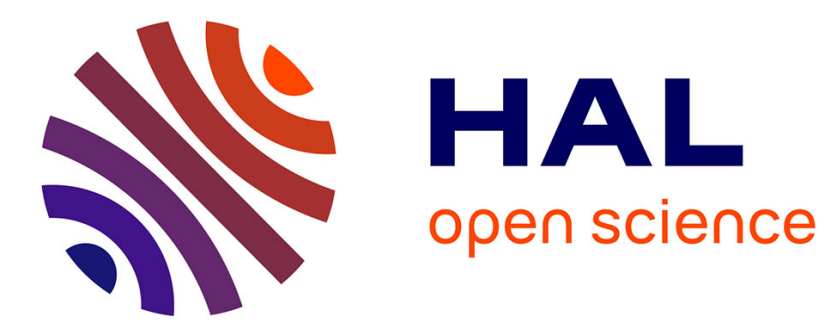

\title{
Expression level of GRP78/BiP as a predictor of favorable or unfavorable outcomes in cancer patients
}

Cyril Ronco, Stéphane Rocchi, Rachid Benhida

\section{To cite this version:}

Cyril Ronco, Stéphane Rocchi, Rachid Benhida. Expression level of GRP78/BiP as a predictor of favorable or unfavorable outcomes in cancer patients. Mediastinum, 2018, 2, pp.26-26. 10.21037/med.2018.03.18 . hal-02415753

\section{HAL Id: hal-02415753 \\ https://hal.science/hal-02415753}

Submitted on 17 Dec 2019

HAL is a multi-disciplinary open access archive for the deposit and dissemination of scientific research documents, whether they are published or not. The documents may come from teaching and research institutions in France or abroad, or from public or private research centers.
L'archive ouverte pluridisciplinaire HAL, est destinée au dépôt et à la diffusion de documents scientifiques de niveau recherche, publiés ou non, émanant des établissements d'enseignement et de recherche français ou étrangers, des laboratoires publics ou privés. 


\title{
Expression level of GRP78/BiP as a predictor of favorable or unfavorable outcomes in cancer patients
}

\author{
Cyril Ronco $^{1}$, Stephane Rocchi ${ }^{2}$, Rachid Benhida $^{1}$ \\ ${ }^{1}$ Institut de Chimie de Nice UMR7272, Université Côte d'Azur, CNRS, 06108 Nice, France; ${ }^{2}$ Centre Méditerranéen de Médecine Moléculaire, \\ Université Côte d'Azur, UMR INSERM U1065, 06204 Nice, France \\ Correspondence to: Cyril Ronco. Institut de Chimie de Nice UMR7272, Université Côte d'Azur, CNRS, 06108 Nice, France. Email: Cyril.ronco@ \\ unice.fr; Stephane Rocchi. Centre Méditerranéen de Médecine Moléculaire, Université Côte d'Azur, UMR INSERM U1065, 06204 Nice, France. \\ Email: stephane.rocchi@unice.fr; Rachid Benhida. Institut de Chimie de Nice UMR7272, Université Côte d'Azur, CNRS, 06108 Nice, France. \\ Email: rachid.benhida@unice.fr. \\ Provenance: This is an invited Editorial commissioned by Section Editor Dr. Zhuoqi Jia (Thoracic Department, the first affiliated Hospital of Xi'an \\ Jiaotong University, Xi'an, China). \\ Comment on: Miura Y, Kaira K, Sakurai R, et al. High expression of GRP78/BiP as a novel predictor of favorable outcomes in patients with advanced \\ thymic carcinoma. Int J Clin Oncol 2017;22:872-879.
}

Received: 02 November 2017; Accepted: 12 March 2018; Published: 30 March 2018.

doi: $10.21037 /$ med.2018.03.18

View this article at: http://dx.doi.org/10.21037/med.2018.03.18

The article "High expression of GRP78/BiP as a novel predictor of favorable outcomes in patients with advanced thymic carcinoma" recently published in the International Fournal of Clinical Oncology describes GRP78/BiP as a marker of favorable outcomes in a particular cancer: advanced thymic carcinoma, a statement a priori in opposition to most of the results published on this topic. Although the methods and protocols used in this article seem coherent and conscientiously carried out, one should first underline the very small size of the patients' cohorts, which hinders high statistic accuracy on the results obtained. Despite the difficulty to gather larger cohorts of patients, due to the inherent rarity of this disease, it would be highly beneficial to confirm these results on a broader population. This once stated, and in spite of a majority of converse reports in other cancers like gastric cancer, breast cancer, prostate cancer, renal cell carcinoma and others, the possibility of obtaining such results cannot be ruled out, but it is crucial to deeply study various parameters before concluding. This editorial aims to help deciphering these different parameters and how they can affect the results and conclusions in clinical studies.

$78 \mathrm{kDa}$-glucose-related protein (GRP78), also named binding immunoglobulin protein $(\mathrm{BiP})$, is a major endoplasmic reticulum (ER) chaperone that belongs to the heat shock protein (HSP)70 family (1). GRP78 is induced by physiologic stress that perturbs ER function and homeostasis, such as glucose deprivation, hypoxia, or poor regulation of calcium concentration. Under these stress conditions, the proteins synthesized by the ribosomes cannot adopt proper secondary structures, and this results in an accumulation of non-/misfolded proteins $(2,3)$. At high concentrations, these malformed proteins aggregate and are toxic for the cell. As a chaperone, GRP78 helps folding proteins in the ER and therefore plays a protective role against cellular stress. In addition to its chaperone function, GRP78 is also the main sensor and activator of a general adaptive mechanism known as Unfolded Protein Response (UPR). UPR aims at resolving the ER stress and is organized in three axes: (1) the attenuation of protein translation in order to slow down the production of proteins, (2) the recruitment of new chaperones (including GRP78 itself) to support the global folding effort and decrease the stock of misfolded proteins, and (3) the evacuation of irrecoverable proteins for their destruction by the proteasome. Two outcomes are then possible for the cell: either the UPR manages to restore ER homeostasis and UPR stops; or the stress is prolonged and cannot be resolved in due time, and UPR triggers apoptosis of the stressed cell (4-7).

UPR plays a critical role in cancer cells which are in an inherent state of stress. Indeed, the protein synthesis 
overactivation to support the intense cell division required for rapid proliferation and the acidic and hypoxic conditions in cancer cells produce a large number of protein folding abnormalities. Therefore, the effect of UPR in cancer may appear at first sight ambiguous. On the one hand, UPR displays a protective role for cancer cells by helping them to survive under stress conditions, but on the other hand it protects also the entire organism by eliminating the dysfunctional too stressed cells $(7,8)$.

There is a difference in GRP78 expression and localization between cancerous and non-cancerous cells. GRP78 expression is maintained at low basal level in major adult organs, while it has been reported overexpressed in many tumors (8-11). Moreover, cancer cells-contrary to normal cells-have been shown to translocate GRP78 at their cell surface, where it is believed to act as a receptor regulating oncogenic signaling, cell viability and angiogenesis in the tumor microenvironment $(10,12)$. Thus it has been logically proposed to target GRP78 to specifically affect the cancer cells, and later to correlate GRP78 cell levels with potential benefic therapeutic outcomes for cancer patients.

However, while accumulating data are available on the role and mechanisms of action of GRP78 in cancer cells, its value as predictive biomarker for disease evolution and prognosis in different cancers is today still debated and some apparently controversy studies have been recently published (13).

GRP78 has been correlated with cancer cell survival, tumor growth, metastasis, tumor size, treatment resistance, protective role against lysis by immune cells, high disease clinical stage, cancer recurrence and low survival (13-15). Furthermore, a poor prognosis has been established for patients with high expression of GRP78 in gastric cancer, breast cancer, prostate cancer, and renal cell carcinoma. On the contrary, several studies report favorable prognosis and higher survival for patients upregulating GRP78 in esophageal, colorectal, lung and thymic cancers (13).

Therefore, to investigate the effects of GRP78 expression, it is important to specify which question is actually addressed and which model is used. Indeed, beyond the apparent paradox of UPR and GRP78 targeting, dosing in vitro the expression levels of GRP78 in cells is different than measuring it in a tumor or an entire organ, studying the impact on drug resistance or evaluating the correlation with a positive prognosis for the patient.

GRP78 localization: GRP78 resides principally inside the lumen of ER, but is also present at the ER membrane alone or with other transmembrane proteins where it may indirectly modulate the activity of proapoptotic components like BH-3, BIK and BAX $(16,17)$. Moreover, GRP78 translocates to the outer membrane of cancer cells where it has shown to play a role in proliferation and cell survival via the activation of PI3K/Akt signaling pathways $(12,18,19)$, and in angiogenesis promotion in the tumour environment. Thus, depending on the GRP78 localization, there might be diverse or opposite inductions of proapoptotic and prosurvival signals. The study of the ratio of these different GRP78 fractions would be particularly interesting to systematically evaluate.

Time-dependent expression: a major parameter that should be taken into account is the kinetics of GRP78 expression. Indeed, GRP78 is the main actor of a balanced homeostatic system that evolves over time. For example, the inhibition of GRP78 leads to ER stress because of the inhibition of its chaperone function and consequently triggers UPR. As a result of UPR activation, GRP78 is over expressed via ATF6 and IRE1 signaling pathways to attempt resolving the ER stress (1). Depending on time and UPR stage, GRP78 expression levels may therefore vary. GRP78 expression has also been proved to differ depending on the disease stage. In esophageal cancer, patients showed upregulated GRP78 in early and late stages, but not in middle stages. The authors speculate a role in local tumor growth control in the early stages and a consequence of high ER stress in the late stages (20). A careful evaluation of GRP78 expression kinetics within the cell and in vivo in patient's tumors would greatly improve our understanding of GRP78 regulation.

Cancer type, histology and micro-environment: the mechanistic functioning differences among various cancer cell types are yet well-known. Another reason that explains the difficulty to obtain homogeneous conclusions is the report of results from different types of cancerous tissues. High GRP78 expression has been shown to be associated with favorable prognosis in esophageal, colorectal, lung and thymic cancers whereas it seems detrimental in gastric, breast, prostate cancer, and renal cancers (13). Moreover, the tumour microenvironment is not homogeneous and presents for example different levels of hypoxia (21). GRP78 is known to be induced by hypoxic conditions, variations in GRP78 expression may be measured inside within the tumour. The differentiation state of the cells might also be taken into account as GRP78 levels might be different in primary cells. A systematic study of GRP78 levels on many various cell lines and tumor tissues would be highly beneficial for a better comparison of its expression. 
Drug resistance: GRP78 has been reported to be overexpressed in resistant cells (to adenoviruses or chemotherapeutic drugs) (22), and therefore to be a potential target to circumvent or re-sensitize cancer cells to standard treatments. However, tumor cell resistance is a complex process that expresses itself simultaneously at different levels and which is highly drug-dependent. If some first line mechanisms are nonspecific and encountered with different drugs like efflux pumps, most of the resistance phenomena rely on specific defenses related to the signaling pathways or to the molecular target of each defined drug. Moreover, most chemotherapeutic drugs enhance cellular stress whether by damaging DNA or by other mechanisms. Therefore, to collect significant data, it is crucial to compare GRP78 levels in patients without treatment (which is rare), or at least tumors from patients receiving the same treatment.

From UPR paradox to in vivo diagnostic tool: beyond all these features remains the inherent paradox of UPR targeting, and the ability to derive from our understanding of these in vitro models an effective diagnostic tool for patients. In cancer, the inhibition of UPR components abrogates the cellular adaptation of cancer cells. On the other hand, ER stress induction leads to sustained UPR activation which leads cells to death (23). Thus, in both cases, by inhibiting it or overloading it, proapoptotic signals can be triggered and the faith of cancer cells is in fact in balance between the prosurvival and proapoptotic branches of the UPR (18). The combined use of drugs could help tilt this balance towards death events. For example, the concomitant use of a repressor of GRP78 expression (like versipelostatin) and of a GRP78 inhibitor might be particularly efficient.

Accumulating experimental data show that UPR targeting drugs promote cancer cells death in vitro and tumor growth inhibition in xenografts models. However, deriving from these results an efficient diagnostic tool for patients is a much more complex question to address that certainly requires more detailed studies. The heterogeneity among cancer cells constrains probably to study case by case GRP78 levels depending on cancer cell type, differentiation status, and micro-environment. The time-dependency of such a kinetic homeostatic system like UPR is also a crucial factor, with GRP78 levels varying during the stages of the UPR response. GRP78 localization and translocation needs also additional understanding, especially its time and cancer type dependence. In patients, the interaction of membrane GRP78 with the immune system cells also requires deeper insight, as this might have a preponderant effect in vivo in patients. Finally, the concomitant use of chemotherapeutic drugs that might directly or indirectly modulate GRP78 levels and expression renders the problem even more complicated, particularly because of the heterogeneity and lack of data concerning the drug regimens of the patients analyzed. Therefore, a combination of all these factors might explain the different conclusions reported in the literature, and additional studies including these parameters will be required in the future to get a better understanding of the correlation between GRP78 levels and disease prognosis for a defined cancer.

\section{Acknowledgements}

GRP78/BiP project was supported by CNRS, INSERM, University of Nice Sophia-Antipolis, INSERM Transfert (COPOC grant), Canceropole PACA (Emerging projects) and ARC (contract n ${ }^{\circ}$ SFI 20121205378).

\section{Footnote}

Conflicts of Interest: The authors have no conflicts of interest to declare.

\section{References}

1. Yoshida H. ER stress and diseases. FEBS J 2007;274:630-58.

2. Healy SJ, Gorman AM, Mousavi-Shafaei P, et al. Targeting the endoplasmic reticulum-stress response as an anticancer strategy. Eur J Pharmacol 2009;625:234-46.

3. Kroemer G, Pouyssegur J. Tumor cell metabolism: cancer's Achilles' heel. Cancer Cell 2008;13:472-82.

4. Lin JH, Li H, Yasumura D, et al. IRE1 signaling affects cell fate during the unfolded protein response. Science 2007;318:944-9.

5. Fribley A, Zhang K, Kaufman RJ. Regulation of apoptosis by the unfolded protein response. Methods Mol Biol 2009;559:191-204.

6. Hetz C. The unfolded protein response: controlling cell fate decisions under ER stress and beyond. Nat Rev Mol Cell Biol 2012;13:89-102.

7. Li X, Zhang K, Li Z. Unfolded protein response in cancer: the physician's perspective. J Hematol Oncol 2011;4:8.

8. Lee AS. GRP78 induction in cancer: therapeutic and prognostic implications. Cancer Res 2007;67:3496-9.

9. Roller C, Maddalo D. The Molecular Chaperone GRP78/ $\mathrm{BiP}$ in the development of chemoresistance: mechanism 
and possible treatment. Front Pharmacol 2013;4:10.

10. Shenolikar S. Dangerous liaisons: flirtations between oncogenic BRAF and GRP78 in drug-resistant melanomas. J Clin Invest 2014;124:973-6.

11. Wang M, Kaufman RJ. The impact of the endoplasmic reticulum protein-folding environment on cancer development. Nat Rev Cancer 2014;14:581-97.

12. Luo B, Lee AS. The critical roles of endoplasmic reticulum chaperones and unfolded protein response in tumorigenesis and anticancer therapies. Oncogene 2013;32:805-18.

13. Miura Y, Kaira K, Sakurai R, et al. High expression of GRP78/BiP as a novel predictor of favorable outcomes in patients with advanced thymic carcinoma. Int J Clin Oncol 2017;22:872-9.

14. $\mathrm{Fu} \mathrm{W,} \mathrm{Wu} \mathrm{X,} \mathrm{Li} \mathrm{J,} \mathrm{et} \mathrm{al.} \mathrm{Upregulation} \mathrm{of} \mathrm{GRP78} \mathrm{in} \mathrm{renal}$ cell carcinoma and its significance. Urology 2010;75:603-7.

15. Jamora C, Dennert G, Lee AS. Inhibition of tumor progression by suppression of stress protein GRP78/BiP induction in fibrosarcoma B/C10ME. Proc Natl Acad Sci U S A 1996;93:7690-4.

16. Ranganathan AC, Zhang L, Adam AP, et al. Functional coupling of p38-induced up-regulation of BiP and activation of RNA-dependent protein kinase-like endoplasmic reticulum kinase to drug resistance of dormant carcinoma cells. Cancer Res 2006;66:1702-11.

doi: 10.21037/med.2018.03.18

Cite this article as: Ronco C, Rocchi S, Benhida R. Expression level of GRP78/BiP as a predictor of favorable or unfavorable outcomes in cancer patients. Mediastinum 2018;2:26.
17. Fu Y, Li J, Lee AS. GRP78/BiP inhibits endoplasmic reticulum BIK and protects human breast cancer cells against estrogen starvation-induced apoptosis. Cancer Res 2007;67:3734-40.

18. Wang M, Wey S, Zhang Y, et al. Role of the unfolded protein response regulator GRP78/BiP in development, cancer, and neurological disorders. Antioxid Redox Signal 2009;11:2307-16.

19. Zhang LH, Zhang X. Roles of GRP78 in physiology and cancer. J Cell Biochem 2010;110:1299-305.

20. Langer R, Feith M, Siewert JR, et al. Expression and clinical significance of glucose regulated proteins GRP78 (BiP) and GRP94 (GP96) in human adenocarcinomas of the esophagus. BMC Cancer 2008;8:70.

21. Martin AR, Ronco C, Demange L, et al. Hypoxia inducible factor down-regulation, cancer and cancer stem cells (CSCs): ongoing success stories. MedChemComm 2017;8:21-52.

22. Tanimoto R, Sakaguchi M, Abarzua F, et al. Downregulation of $\mathrm{BiP} / \mathrm{GRP} 78$ sensitizes resistant prostate cancer cells to gene-therapeutic overexpression of REIC/ Dkk-3. Int J Cancer 2010;126:1562-9.

23. Vandewynckel Y-P, Laukens D, Geerts A, et al. The paradox of the unfolded protein response in cancer. Anticancer Res 2013;33:4683-94. 